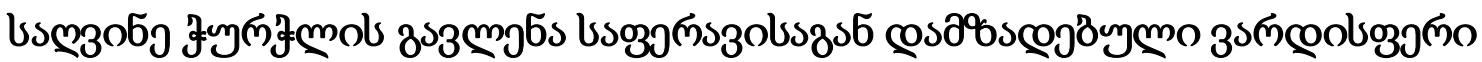 œ3o6ol bsmolb\%?
}

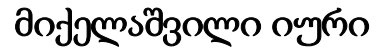

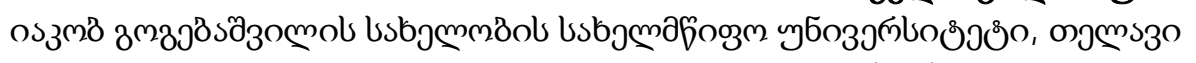

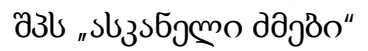

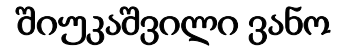

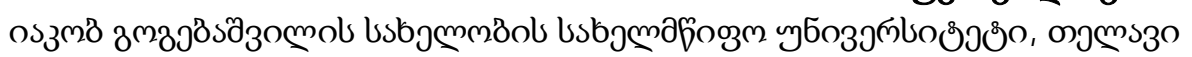

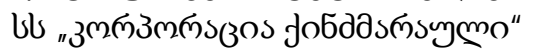 \\ 3ээbодзомо бобм
}

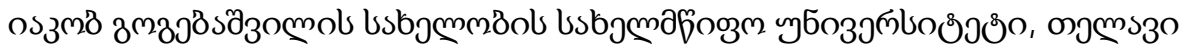

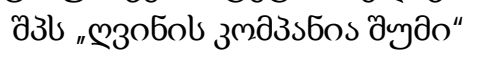

https://doi.org/10.52340/idw.2021.497

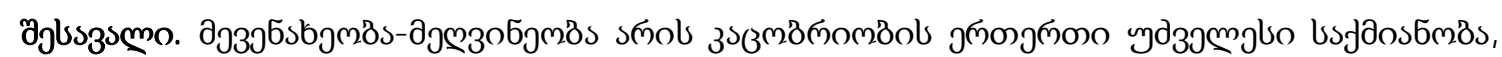

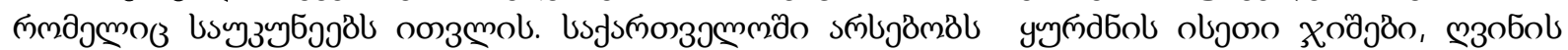

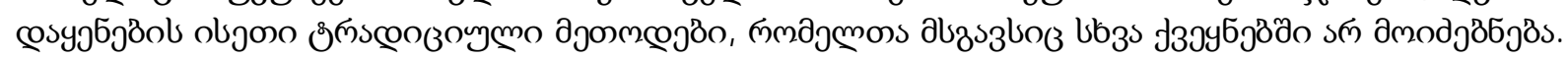

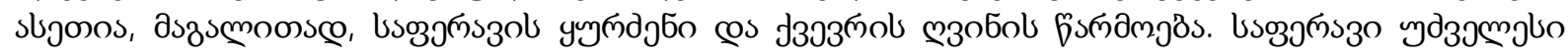

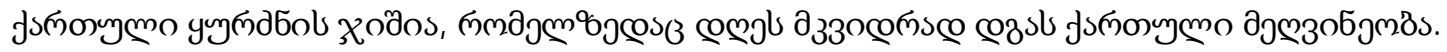

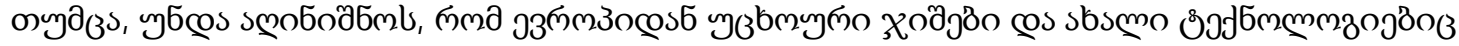

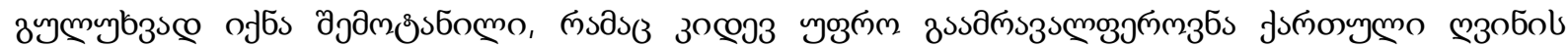

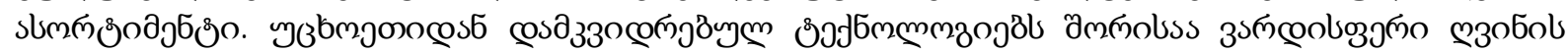

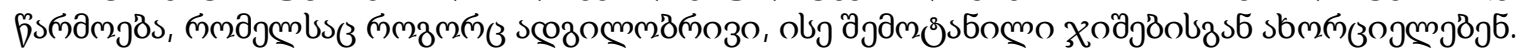

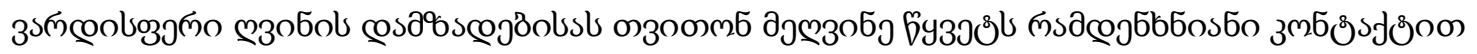

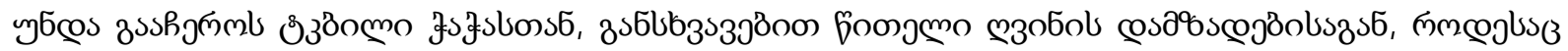

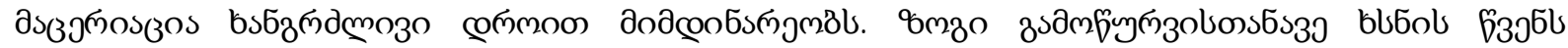

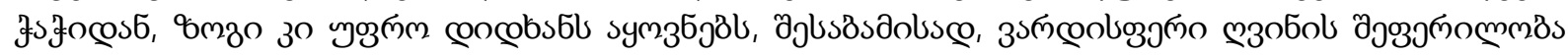

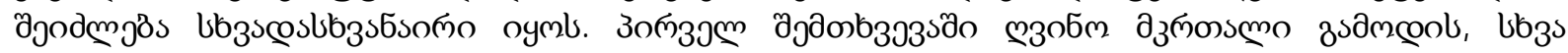

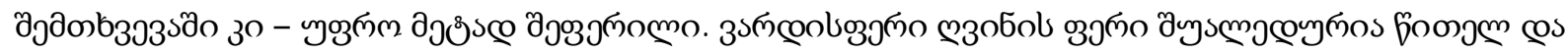

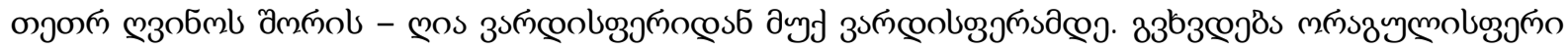

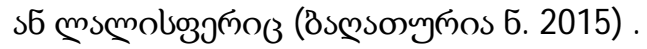

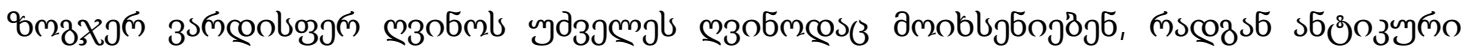

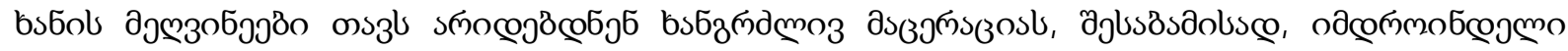

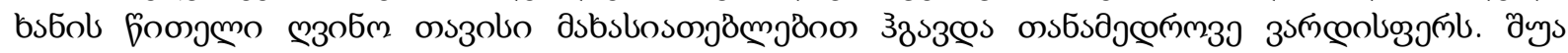

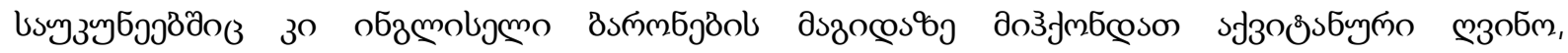

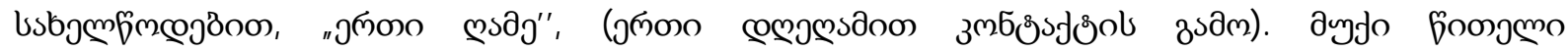

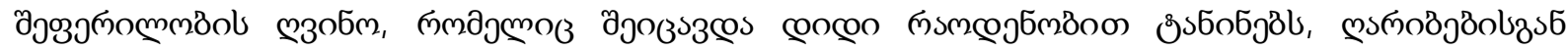

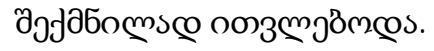

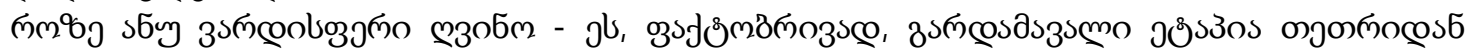
foom

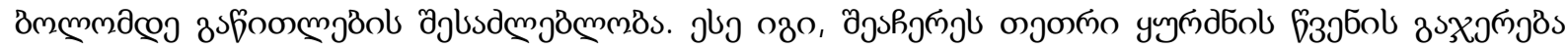

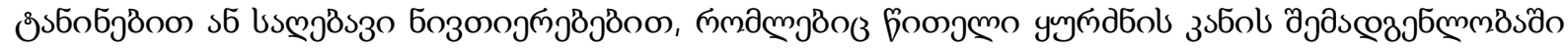
gocol.

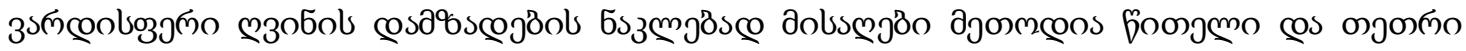

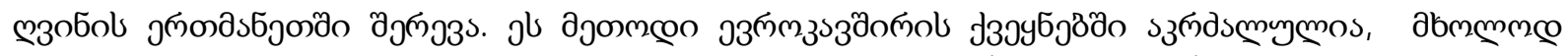

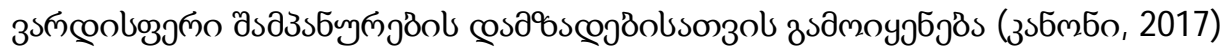




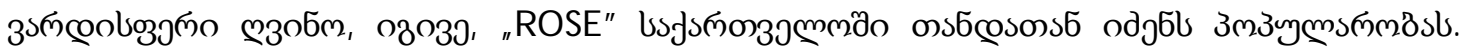

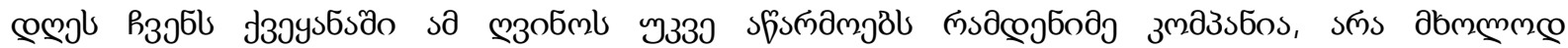

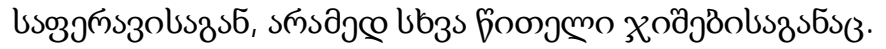

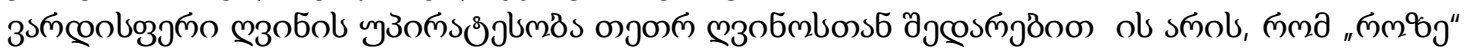

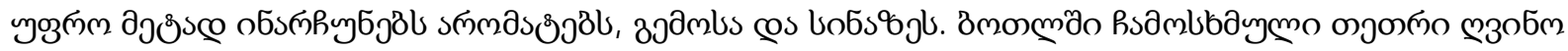

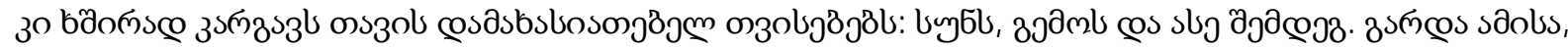

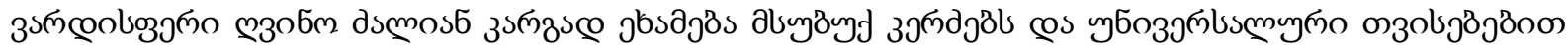

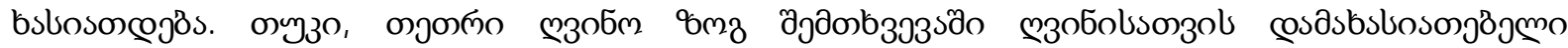

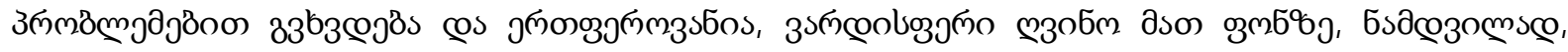

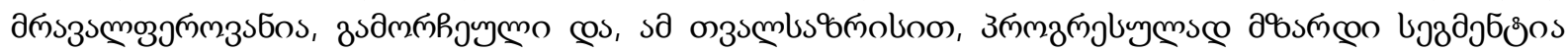

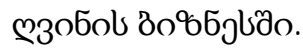

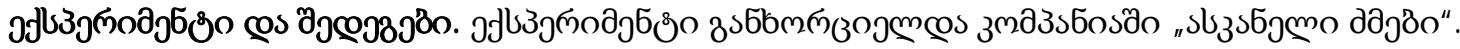

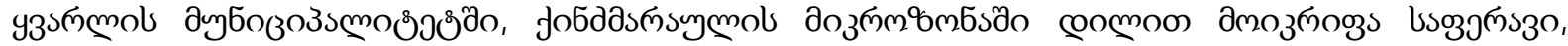
дј๓

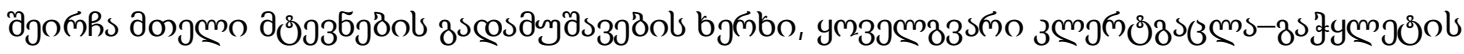

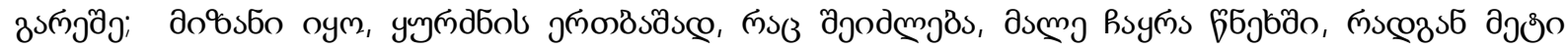

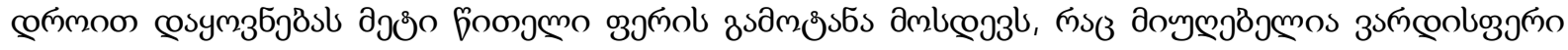
sзoбolomzol.

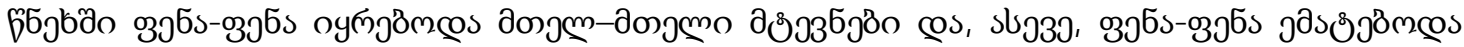

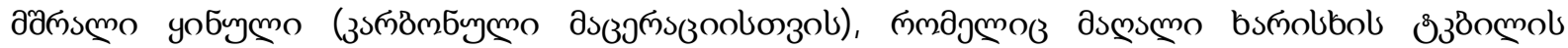

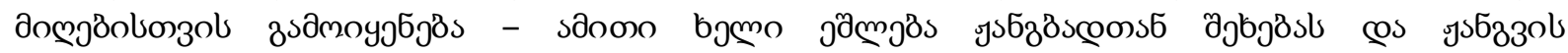
sলিslsblum

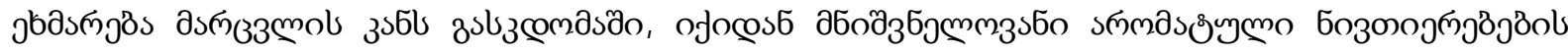

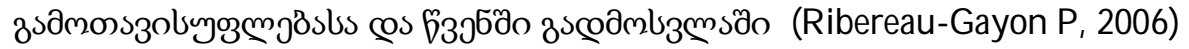

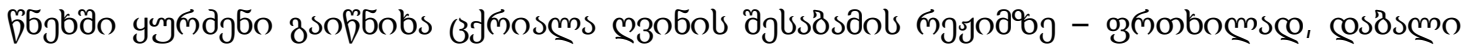

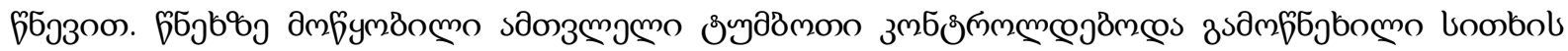

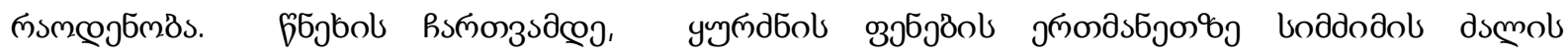

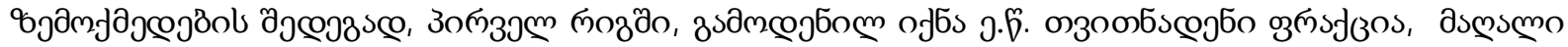

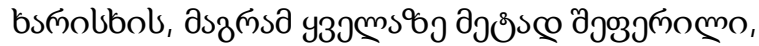

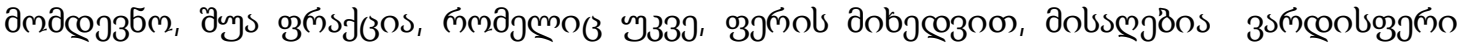

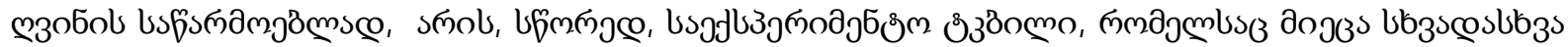

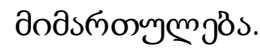

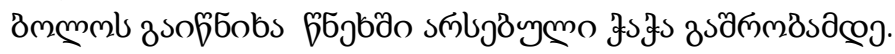

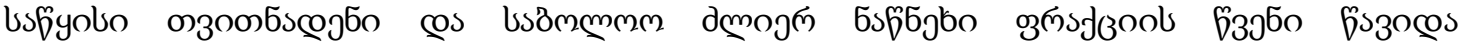

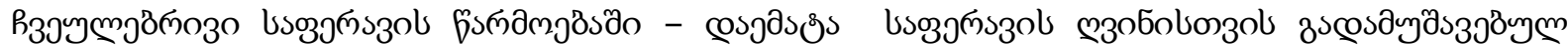

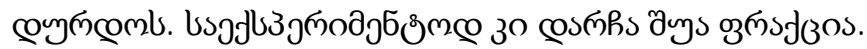

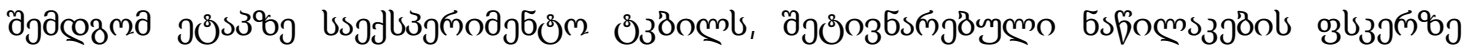

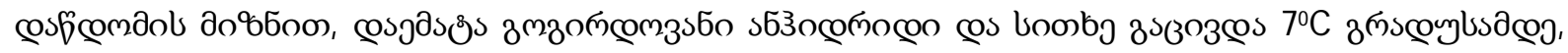

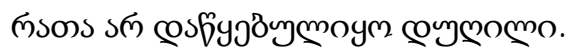

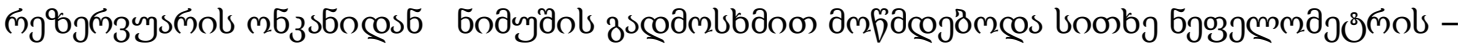

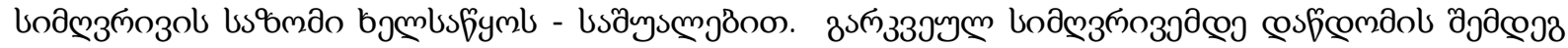

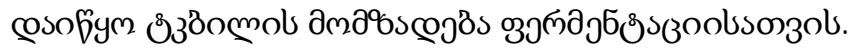

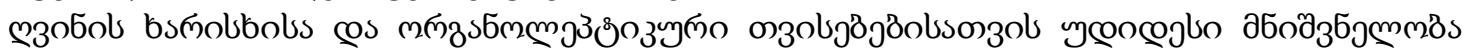

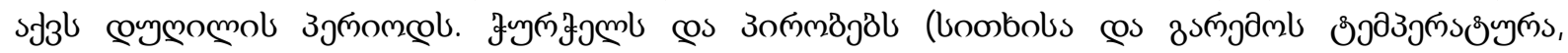

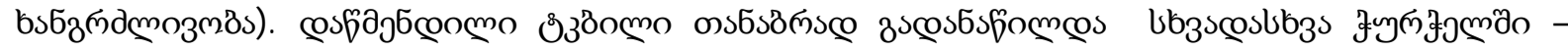

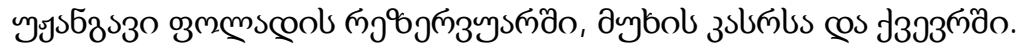

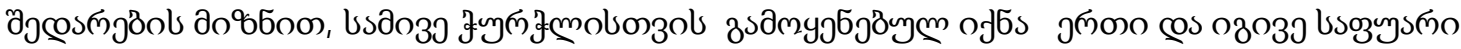
B-2000 @os $\partial_{33 J^{8}} 83_{3}$ GO-FERM PROTECT

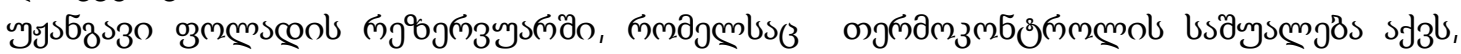

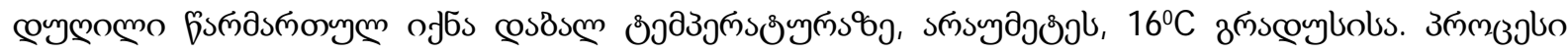




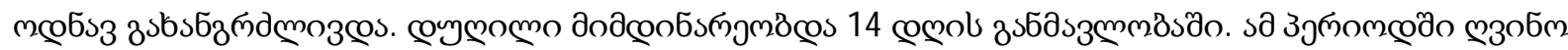

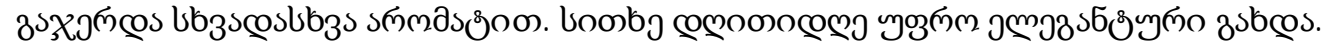

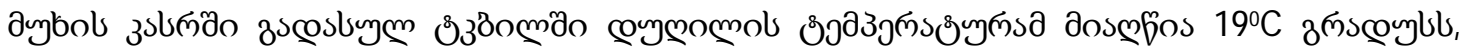

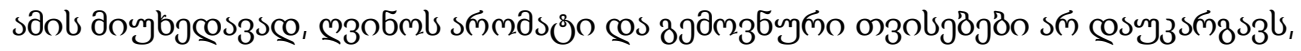

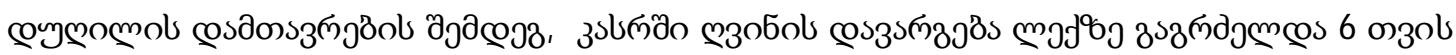

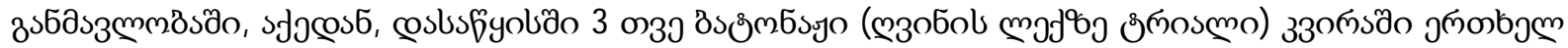

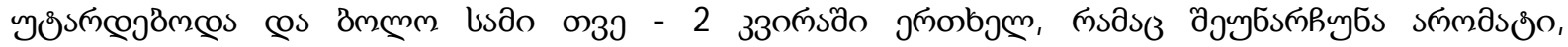

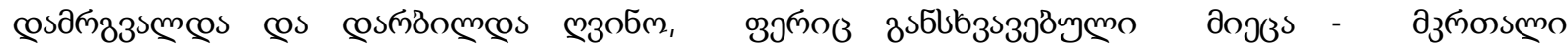

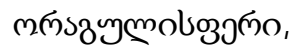

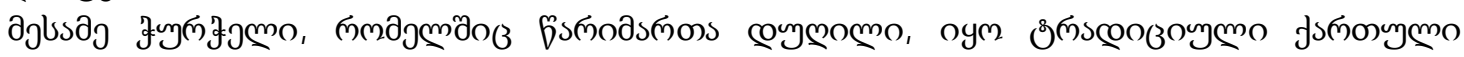

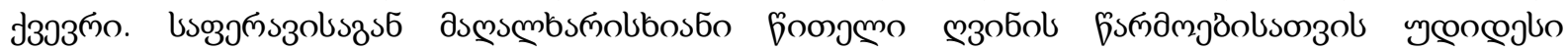

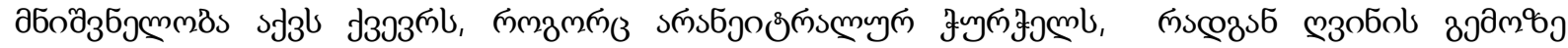

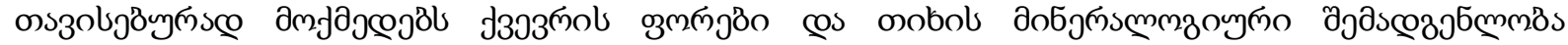

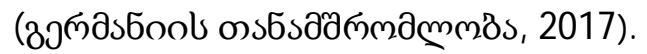

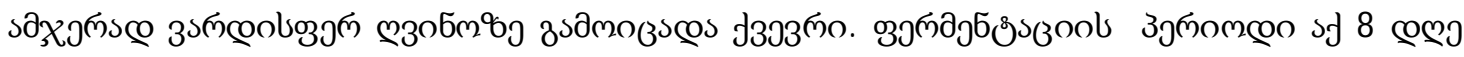

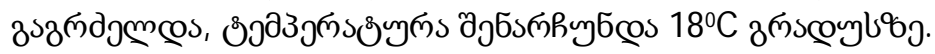

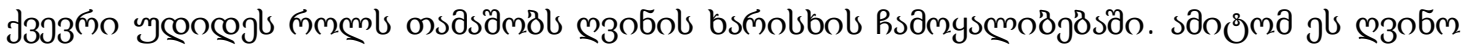

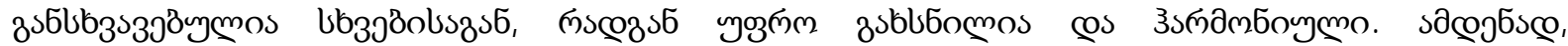

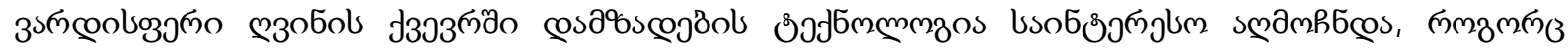

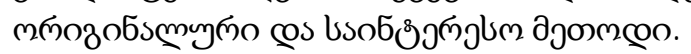

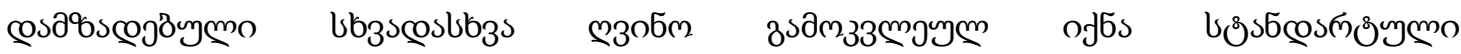

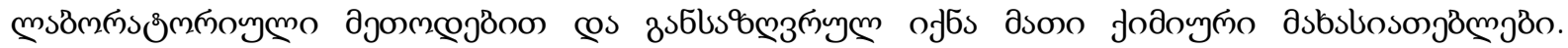

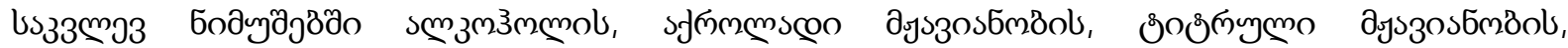

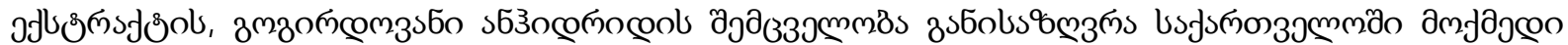

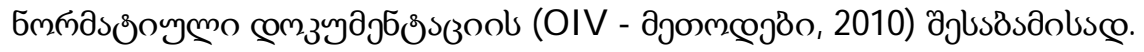

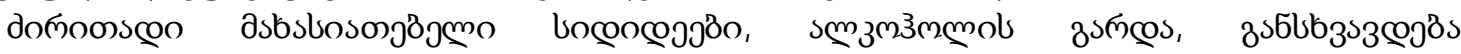

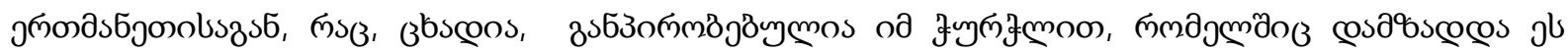

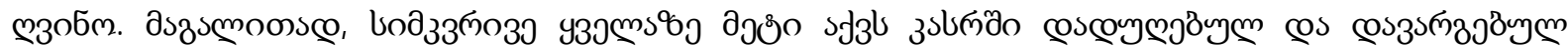

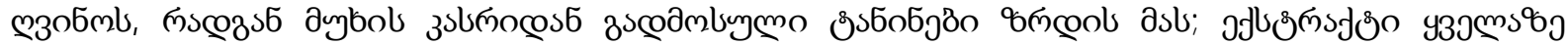

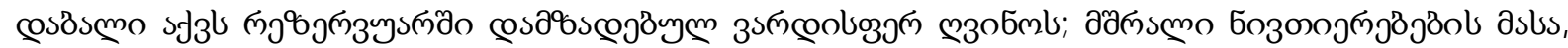

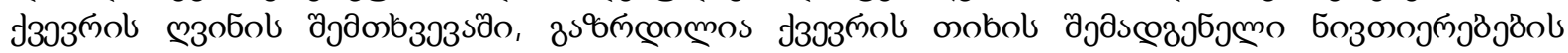

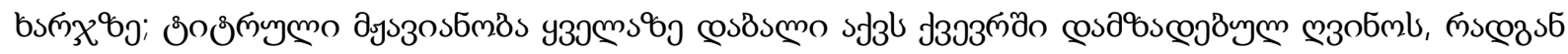

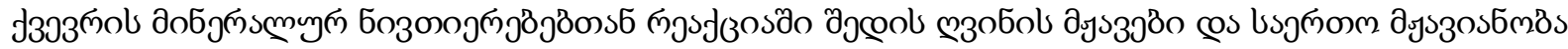

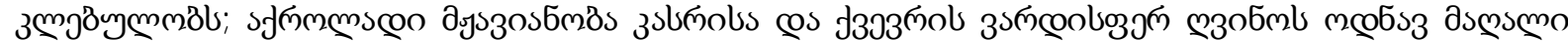

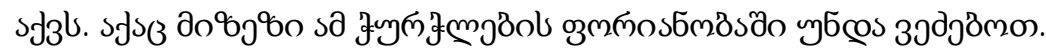

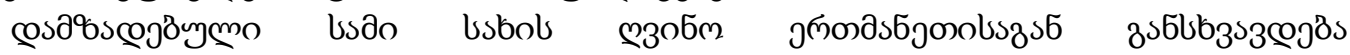

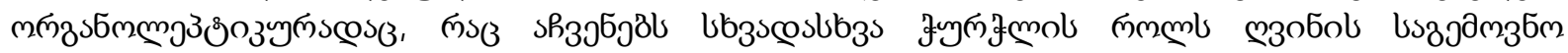

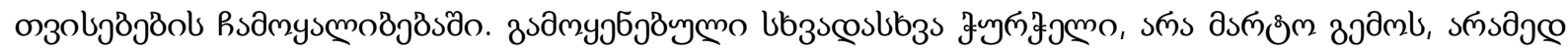

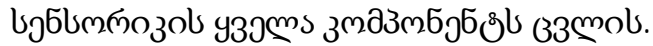

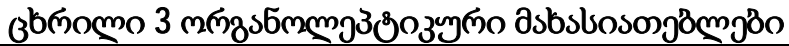

\begin{tabular}{|c|c|c|c|}
\hline 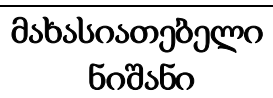 & 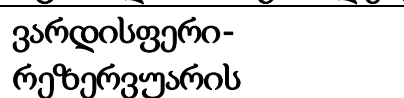 & 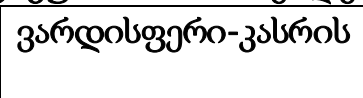 & 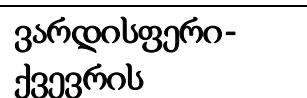 \\
\hline зวॅпо & 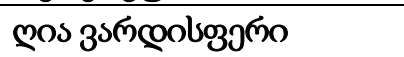 & 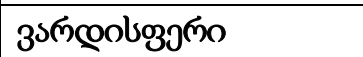 & мஙু \\
\hline$\partial \partial^{\partial \mathrm{m}}$ & 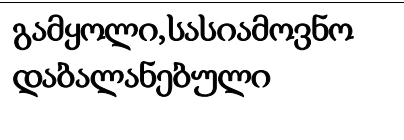 & 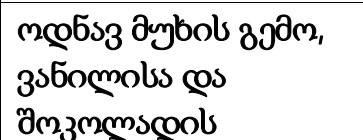 & 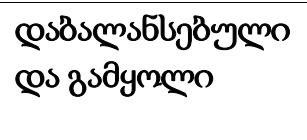 \\
\hline ১ங்றపుతిం & 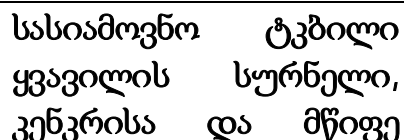 & 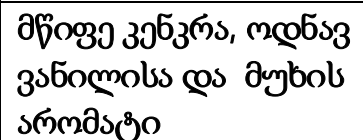 & 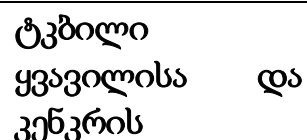 \\
\hline
\end{tabular}




\begin{tabular}{|c|c|c|c|}
\hline & 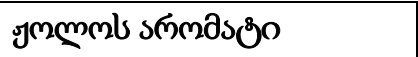 & & вмупбомо \\
\hline Зృґдамбоумммдे & 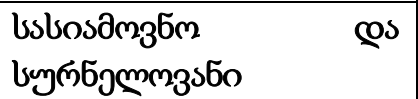 & мебзз дмзущучмо & $\begin{array}{l}\text { bsmolostoo cos } \\
\text { asblb6omo }\end{array}$ \\
\hline
\end{tabular}

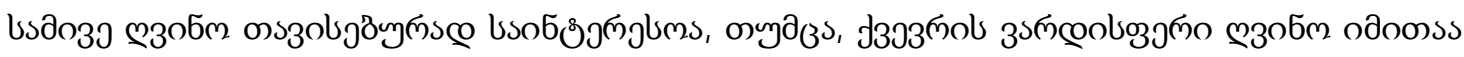

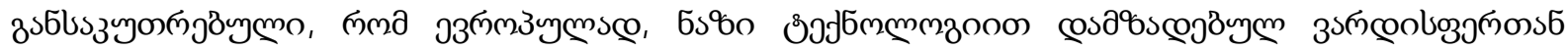

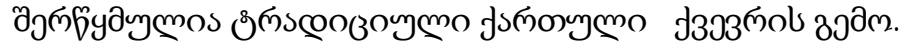

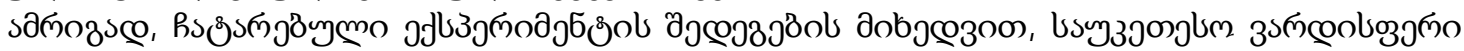

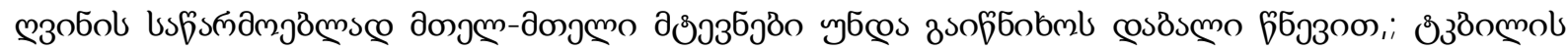

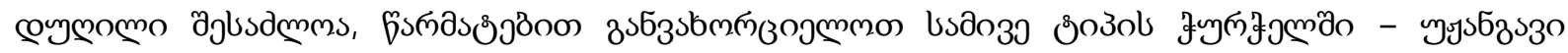

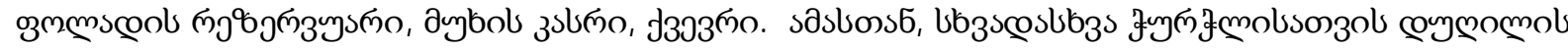

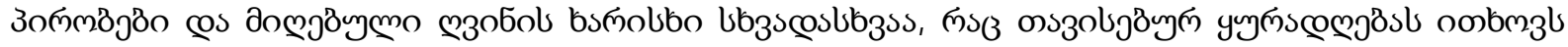

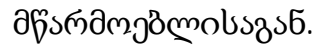

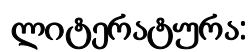

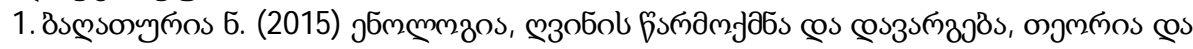

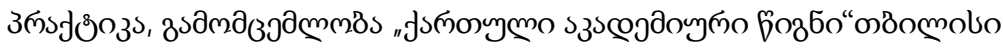

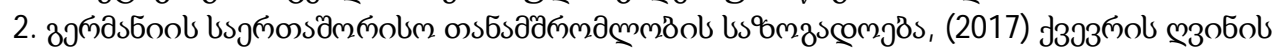
о@jб

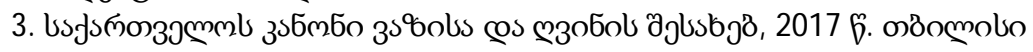

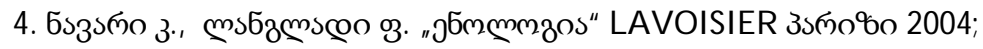

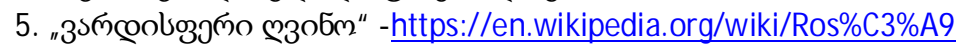

6. O.I.V - 2010 Office International De La Vigne Et Du Vin, Recueil des methods internationales d'analyse des vins. Paris,;

7. Ribereau-Gayon P,, Dubourdieu D. "The Microbiology of Wine and Vinifications $2^{\text {nd }}$ Edition" Copyright 2006 John W iley \& Sons Ltd, The A trium, Southern Gate, Chichester, W est Sussex P019 8SQ, England;

\section{Impact of wine vessels on the properties of rose wine made from Saperavi}

\author{
Mikelashvili Iuri \\ Iakob Gogebashvili State University, Telavi \\ Ltd. "A skaneli Brothers" \\ Shiukashvili Vano \\ Iakob Gogebashvili State University, Telavi \\ JSC "Corporation Kindzmarauli" \\ Vephkhishvili Nino \\ Iakob Gogebashvili State University, Telavi \\ Ltd. "W ine Company Shumi"
}

\begin{abstract}
Viticulture - winemaking is one of the oldest activities of mankind, which dates back centuries. In Georgia there are such grape varieties and such traditional methods of winemaking that are not found in other countries. For example, Saperavi grape and production of Qvevri (pitcher) wine. Saperavi is an ancient Georgian grape variety on which Georgian winemaking is based today.

We have established experimentally the possibility of making high quality rose wine with various characteristics from Saperavi grown in Kakheti region using different wine vessels, such as stainless steel reservoir, oak barrel, traditional Georgian Qvevri (pitcher), under production
\end{abstract}


conditions. The physical, chemical and organoleptic properties of various wines that provide the individuality of the wine have been determined.

The main quantities characteristic to rose wine differ from one another, which is obviously due to the vessel in which the wine was made. For example, the wine, fermented and aged in the barrel has the most density because the tannins from the oak barrel increase it; the rose wine, made in the reservoir, has the lowest extract; in the case of Qvevri (pitcher) wine, the mass of dry matter is increased at the expense of the constituent substances of the clay of the pitcher; the wine made in the pitcher has the lowest titratable acidity because the wine acids go into the reaction with the mineral substances of the pitcher clay and the overall acidity decreases; the barrel and Qvevri rose wine has slightly higher volatile acidity. Here the reason for that should also be found in the porosity of the barrel and the pitcher.

The various wines produced differ from one another organoleptically as well, which shows the role of the vessel in forming the taste properties of the wine. The different vessels used change all the components of the sensorics.

All three wines are interesting in their own way, but the rose Qvevri wine is special for the taste of the traditional Georgian pitcher is best combined with the rose wine made with European technology and gentle processing.

Key W ords: Saperavi, rose wine, reservoir, barrel, Qvevri (pitcher)

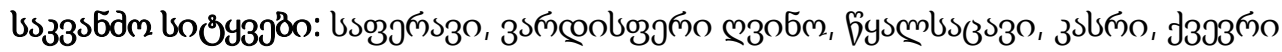

